\title{
Establishment of in vivo - in vitro Correlation: a Cogent Strategy in Product Development Process
}

\author{
Sharwaree Hardikar*, A V Bhosale and R N Budhawant \\ Department of Pharmaceutics, Pune District Education Association's Seth Govind Raghunath Sable College of Pharmacy, Saswad. \\ Tal- Purandhar, Dist.- Pune 412301. India
}

\begin{abstract}
Background: In vivo In vitro correlation (IVIVC) development and its validation is an extremely important phase of dosage form (new as well as generic) optimization. It is a liaison (preferentially linear) between a suitable pharmacokinetic parameter (peak plasma drug conc. i.e. Cmax, time for peak plasma drug conc. i.e. tmax and Area under the Curve i.e. AUC) and in vitro characteristics. Need: During optimization modifications in formulation composition, manufacturing method, required equipments or even in batch size. If these changes are made, in vivo bioavailability study in healthy human volunteers may be required to prove bioavailability of newly developed dosage form with the earlier one. These facts halt the marketing of new formulation with added cost. Thus, it would be enviable to develop in vitro test that corresponds to bioavailability data. Applications: Thus the main purpose of development of IVIVC is to replace in vivo bioavailability and to support bio waivers. As IVIVC comprises linkage between suitable in vivo pharmacokinetic parameter to in vitro characteristic pertaining to dissolution; it can also be used to set up dissolution specifications and/ or validation of methods of dissolution studies. It also assists as means of quality control for certain SUPAC (Scale Up and Post Approval Changes). Moreover there must be some in vitro means to assure batch to batch consistency during large scale manufacturing. After successful establishment of IVIVC; dissolution method used in such establishment is used as routine quality control test as a measure of physiological performance of drug product.
\end{abstract}

Keywords: In vivo In vitro correlation, Biowaiver, Deconvolution, Convolution.

\section{INTRODUCTION}

Formulation development is critical stage of entire product development process. Establishment of In vivo In vitro correlations (IVIVC) is an integral part of formulation development and optimization. The overall tenure involved in momentous institution of IVIVC is depicted in (Figure 1).

IVIVC is a mathematical relationship between in vitro properties (dissolution) of the dosage form to its bioavailability. IVIVC reduces the number of clinical trials required in prop up of regulatory approval of a new product and during SUPAC (Scale-Up and Post Approval Changes). Regulatory guidance was developed by FDA and referred as IVIVC Guidance. ${ }^{1,2}$
Thus, IVIVC plays a key role in product development. ${ }^{1,3}$ As it Serves as a replacement of in vivo and helps to support biowaivers (particularly in case of BCS class I drugs), Use full in selection of dissolution schemes and specifications and their eventual validation, Assists in assessment of quality of the product during manufacturing and Useful in optimizing formulations.

\section{Establishing the Dissolution Specifications}

From the preclinical development until proof of concept initiation for design of dosage form, pharmacokinetic testing is often carried out. Pharmacokinetics of different physical forms, salts, variable particle sizes and more than one formulations of
Submission Date :03-04-14 Revision Date : :16-06-14 Accepted Date : 03-09-14

DOI: 10.5530/ijper.48.4.9

Address for correspondence:

Sharwaree Hardikar Pune District Education Association's Seth Govind Raghunath Sable College of Pharmacy, Saswad. Tal- Purandhar, Dist. Pune 412302.

Phone/Fax: 02115-222212

E-mail: sharwareehardikar@ gmail.com

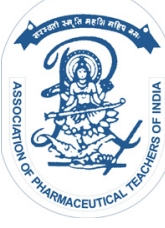

www.ijper.org 


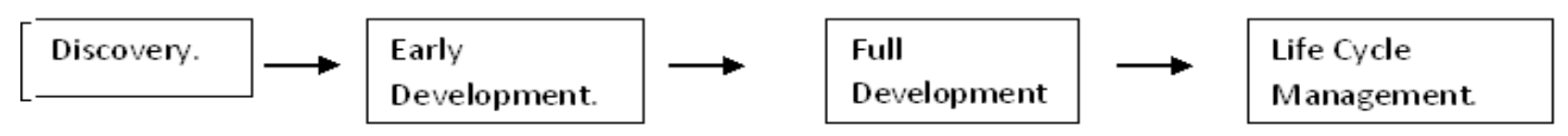

\section{In Silico Simulation}

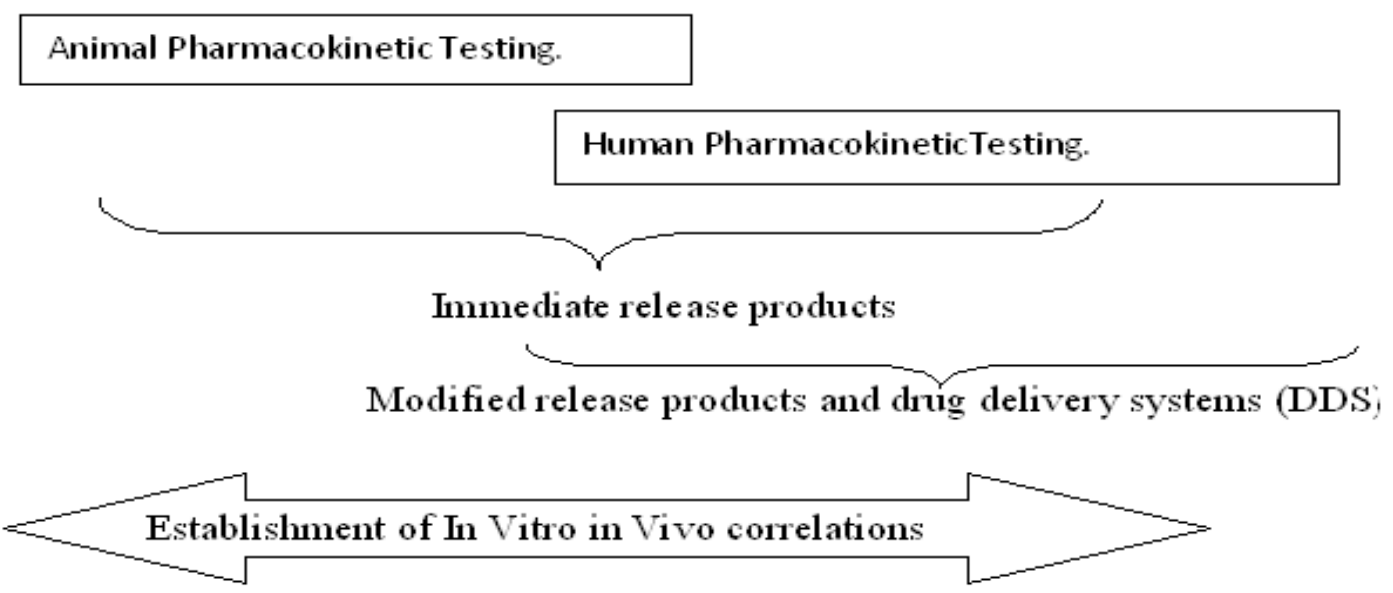

Figure 1: IVIVC in Drug Development Process.

drug molecule can be evaluated in a preclinical animal model. This is the first time when in vitro measurement (i.e. dissolution profile) is correlated to its in vivo performance such as $\mathrm{C}_{\max }$ and Area under the Curve (AUC). At this stage, bio relevant dissolution method is developed. Bio relevant dissolution method involves bio relevant dissolution media that are virtually same as biological fluids. Use of such media assists successful establishment of IVIVC. ${ }^{4,5}$

Generic products are often developed by using new excipient which is different than used in branded product. Not only excipient but manufacturing process may differ significantly. Hence dissolution methods and specifications for branded product may not be suitable for generic products; though they are bioequivalent. A preclinical pharmacokinetic study (bioavailability studies in animal) for such generic products (even for newly developed product) may be used to develop appropriate dissolution method. ${ }^{4}$ IVIVC establishment using human pharmacokinetic data has scientific and practical value. This further supports future formulation development during life cycle management.

Generally dissolution test is employed to assess the impact of formulation and processing variables on drug release characteristics. It is also the primary evaluation parameter that can be correlated with bioavailability of drug under consideration. Thus correlation in some form is desirable between suitable in vitro dissolution characteristics e.g. $t_{50 \%}, t_{90 \%}$ etc. and relevant in vivo pharmacokinetic parameter e.g. $\mathrm{t}_{\max }$; which is referred as IVIVC. Developing a dissolution test with an aim to establish such relationship is an ever challenging job of a formulation development pharmacist/ researcher in the overall development process of a drug product. Biopharmaceutics Classification System (BCS) guides researcher in deciding the terms under which the IVIVCs are anticipated (table 1). A decision tree for dissolution testing method/design based on BCS and physicochemical properties of the molecule have been proposed (Figure 2, 3). ${ }^{6-8}$

\section{Development of in vitro in vivo Correlations}

Tactical development of such relation is two stage programme ${ }^{9}$

IVIVC establishment is a vivacious process and commences from the initial period of product development. Clinical phase trials and formulation development goes contemporarily to save the time involved in overall product development process.

An assumption of IVIVC i.e. appropriate in vitro dissolution targets to be predefined to meet the desired in vivo bioavailability specifications assuming level A. This correlation hypothetical pharmacokinetic/pharmacodynamic model is subject to revision as the prototype formulation is analyzed for in vivo behavior. Usually more than one prototype formulations within one technology are tested with the dissolution methodology and technology available in the laboratory. Thus this activity culminates in a pilot pharmacokinetic study. The results of this pilot pharmacokinetic study result in retrospec- 


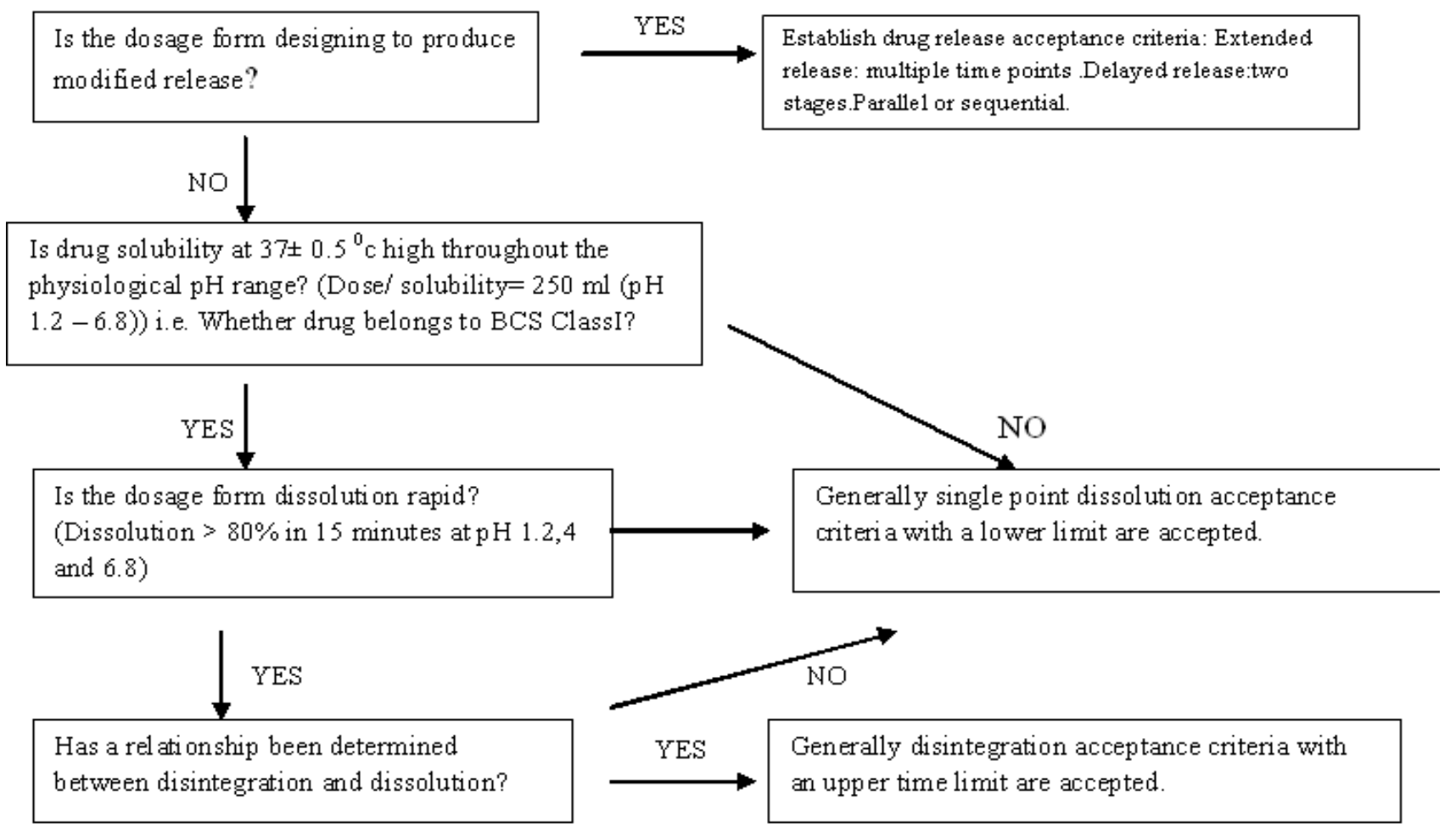

Figure 2. A Decision Tree for Dissolution Testing Based on BCS for Appropriate Type of Drug Release Acceptance Criteria for IVIVC.

\begin{tabular}{|cccc|}
\hline Class & Solubility & Permeability & Dissolution Methodology \\
\hline I & High & High & $\begin{array}{c}\text { Single point if NLT 85\% Q in } 15 \text { min } \\
\text { Multiple point if } Q<85 \% \text { in } 15 \text { min }\end{array}$ \\
\hline II & Low & High & Multiple point \\
\hline III & High & Low & Same as Class I \\
\hline IV & Low & Low & Same as Class II \\
\hline
\end{tabular}

Multiple Point Test: 4 - 6 points each test

Test 1: $\mathrm{pH}=1,2 \mathrm{hr} .$, Volume $=250 \mathrm{ml}$

Test 2: Media Change at $0.5,1,2$ hr. to $\mathrm{pH} \mathrm{4.5,6.5,8.0}$

Surfactant media when required to achieve $\mathrm{Q}=\mathbf{8 5} \%$, Volume $=900 \mathrm{ml}$

Figure 3. Dissolution Methodology for Immediate Release Products.

tive IVIVR. This work many times steers the process of resetting of in vitro target revision of formulation strategy.

Once defined formulation is developed that meets the in vivo target; the next aim is to progress for its approval through optimization. Optimized formulation is one which exhibits drug release profile that reveals mecha- nism of release. A release rate controlling parameter is identified from the retrospective data generated in first stage. In second stage extensive in vitro dissolution study is performed across dissolution media of using variable $\mathrm{pH}$ and various apparatus for optimized formulation. This leads to execution of prospective IVIVR study which is useful further in quality control method devel- 
opment. This is ideally level A IVIVC but multiple levels C IVIVC is acceptable.

Thus a typical IVIVC development involves three essential elements, (i) model development, (ii) model validation and (iii) model application. To develop a meaningful IVIVC depends upon the design of the delivery system, its composition, manufacturing process, properties of the drug and the method of dissolution. ${ }^{5}$

\section{IVIVC Model Development}

Five correlation levels are defined in the FDA guidance viz. IVIVC level A, IVIVC level B, IVIVC level C, IVIVC level D, and IVIVC multiple level C. ${ }^{10}$ The level of correlation is decided from the propensity of IVIVC to reflect the complete plasma drug level-time profile. In the process of IVIVC development, the in vitro drug release parameters which resemble in vivo drug performance are identified. The suitable design of in vitro dissolution tests, capable of differentiating between the formulations with dissimilar bioavailability, plays a key role in the certainty of the IVIVC. Therefore, it is necessary that in vitro dissolution tests precisely reflect in vivo conditions, when they are used to set up an IVIVC.

IVIVC at "Level A" This is the most common type of correlation observed in new drug applications (NDAs) submitted to the FDA. From regulatory perspectives, it is considered to be the most useful. It is the most acceptable level of correlation. This level is accepted by regulatory authorities because of existence of point to point correlation during the time course between in vivo and in vitro profiles. The establishment of a robust Level A correlation involves adaption of either deconvolution or convolution method.

\section{Deconvolution Methods}

For developing IVIVC Level A, that can be accepted by regulatory agencies, an IVIVC model needs to be developed for at least two formulations, with three or more being preferred. The assessment of in vivo absorption contour from the concentration-time data can be achieved through the three stage deconvolution methods involving Wagner-Nelson (WN), Loo-Riegelman (LR) methods or model independent methods. The model-dependent Wagner-Nelson method is derived by adopting one-compartment model; it has an advantage that it demands plasma profile of the drug after oral administration only. The Loo-Riegelman method needs intravenous data and is derived by adopting twocompartment model. In vivo plasma data from an oral solution/intravenous dosing is essential for the application of model-independent numerical deconvolution.

The in vitro dissolution profiles are scaled by mathematical means, as there are intrinsic differences in vitro disso- lution specifications and in vivo biological conditions and represented by equation 1 and is referred to as 'deconvolution method' ${ }^{4}$.

$$
\begin{aligned}
X \text { vivo }(\mathrm{t})= & \mathrm{A}_{1}+\mathrm{A}_{2} \times \mathrm{X} \text { vitro }\left(\mathrm{B}_{1} \times \mathrm{B}_{2} \mathrm{x}\right) \text { if } \mathrm{t}>\mathrm{T} \text { then } \mathrm{t}=\mathrm{T} \\
& \ldots \ldots \ldots \text { Eq. }{ }^{\mathrm{n}} 1 . \\
& \text { if } \mathrm{B}_{1}<\mathrm{B}_{2} \times \mathrm{t} \text { then } \mathrm{B}_{1}+\mathrm{B}_{2} \times \mathrm{t}=0
\end{aligned}
$$

Whereby, $\mathrm{X}$ vivo $(\mathrm{t})$ represents the absorption profile as a function of time and $\mathrm{X}$ vitro $(\mathrm{t})$ represents the dissolution profile. The modifications to the in vitro profile as a function of time $t$ is achieved by introduction of a time scale factor $\mathrm{B}_{2}$. If the in vitro dissolution process occurs faster or slower than the corresponding in vivo dissolution, the correction factors are added. To allow an initial lag time in the in vivo absorption (because of necessary pre absorption transit through the stomach) through a lag time $\mathrm{B}_{1 ;}$ and through a cut-off factor $\mathrm{T}$ to accommodate dissolution slower than the GI transit times. The actual correlation is obtained via comparison of the in vivo profile with the slope $\mathrm{A}_{2}$ and $\mathrm{y}$-intercept $\mathrm{A}_{1}$ as a link function between both profiles.

\section{Determining the Fraction of Dose Absorbed (For determining $X$ vivo $(t)):{ }^{11}$}

1. Wagner Nelson method - This is predictive mathematical equation for establishment of IVIVC of drug following fist order kinetics in one compartment model.

$$
\mathrm{F}_{(\mathrm{t})}=\mathrm{C}_{\mathrm{t}}+\frac{\mathrm{K}_{\mathrm{el}} \mathrm{AUC0} 0^{\mathrm{t}}}{\mathrm{K}_{\mathrm{el}} \mathrm{AUC}_{0}^{\infty}}
$$

Where is fraction bioavailable (absorbed), $\mathrm{C}_{\mathrm{t}}$ is concentration of drug at time $t$ in blood / plasma, drug's elimination rate constant at time $\mathrm{t}$ is $\mathrm{K}_{\mathrm{el}}$ and AUC is Area under the Curve. This equation is useful to transform $C_{t}$ values to values as first step of deconvolution method.

2. Loo-Riegelman method: It is useful in a drugs following two compartment models and obeying first order kinetics.

$$
\mathrm{F}_{(\mathrm{t})}=\frac{\mathrm{C}_{\mathrm{t}+} \mathrm{K}_{\mathrm{el}} \mathrm{AUC}_{(\mathrm{o}-\mathrm{t})}+\left(\mathrm{X}_{\mathrm{p}}\right)_{\mathrm{t}} / \mathrm{V}_{\mathrm{c}}}{\mathrm{K}_{\mathrm{el}} \mathrm{AUC}_{(0-\infty)}}
$$

$\mathrm{Ct}$ and $\mathrm{AUC}_{(\mathrm{O}-\mathrm{t})}$ are calculated from the plot of $\mathrm{Ct}$ vs. $\mathrm{t}$ and the values of $\left(\mathrm{X}_{\mathrm{p}}\right)_{\mathrm{t}} / \mathrm{V}_{\mathrm{c}}$ can be approximated by Loo-Riegelman method. $\mathrm{K}_{\mathrm{el}}$ is elimination rate constant of a drug and AUC is Area under the Curve.

3. Model independent methods or numerical deconvolution method: It is particularly useful when the drug follows nonlinear kinetics.

\section{Convolution Methods: ${ }^{4}$}

Conversion of the dissolution contour to a plasma concentration contour can take place via convolution (input to output). Recently, convolution methods have been 


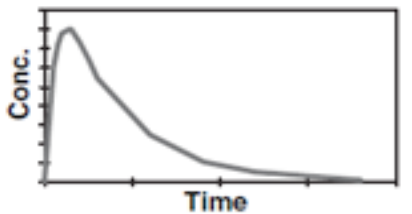

In vivo Plasma Concentration Profile
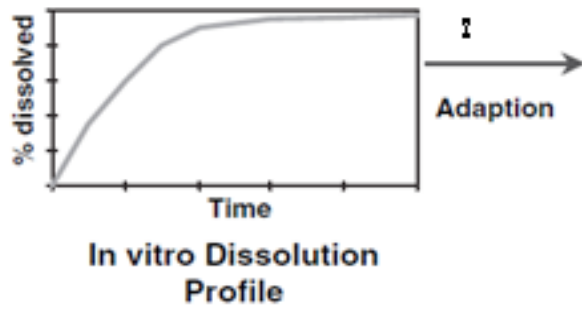

Profile

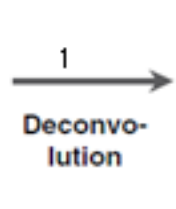

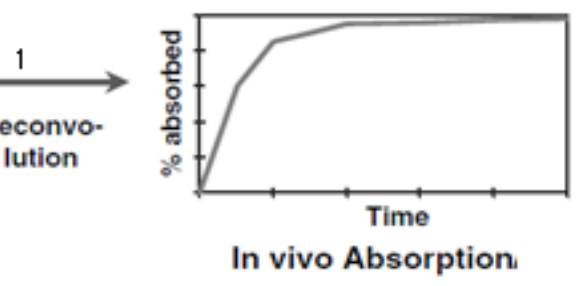

In vivo Absorption
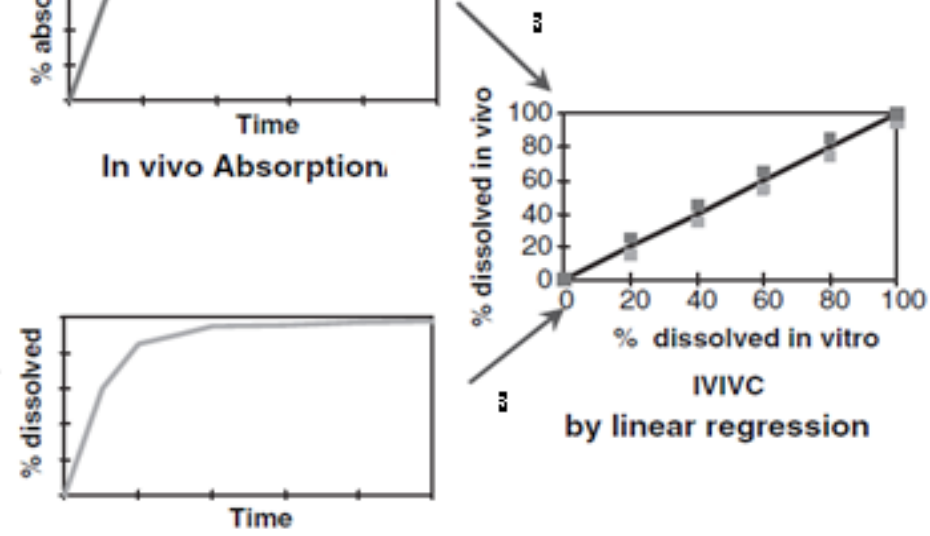

Adapted In vitro Dissolution Profile

Figure 4. Classical Three Steps of Deconvolution.

established, which convolutes the dissolution contour without applying the correlation of the absorption/in vivo dissolution contour with the dissolution profile in vitro (i.e., physiology based model and simulation software). The model uses multiple differential equations representing various physiological events and convolution-based methods. Such a convolution does not take place in several partial stages, rather in one single stage that is schematically illustrated in (Figure 4 and 5). A major advantage of convolution-based methods for IVIVC is that no additional in vivo data such as intravenous injections or oral solutions are required. However, these methods can only mathematically fit the data by minimizing the squared error; even though the results obtained are mathematically correct it may not be meaningful PK or physiological models. A critical assessment of the calculated parameters is absolutely necessary. Further, the fitting procedure should be performed several times with different starting values, in order to avoid reaching a local minimum. Last but not the least, these methods should be optimized to as few variables as possible, as the fitting procedure becomes more complex and error-prone with more variables.

IVIVC at "Level B" If the entire profile cannot be used for correlation, reduction in in vivo and the in vitro data that is comparable is required. The model-independent statistical moment theory uses empirical distribution functions for the purpose. Level B correlation is
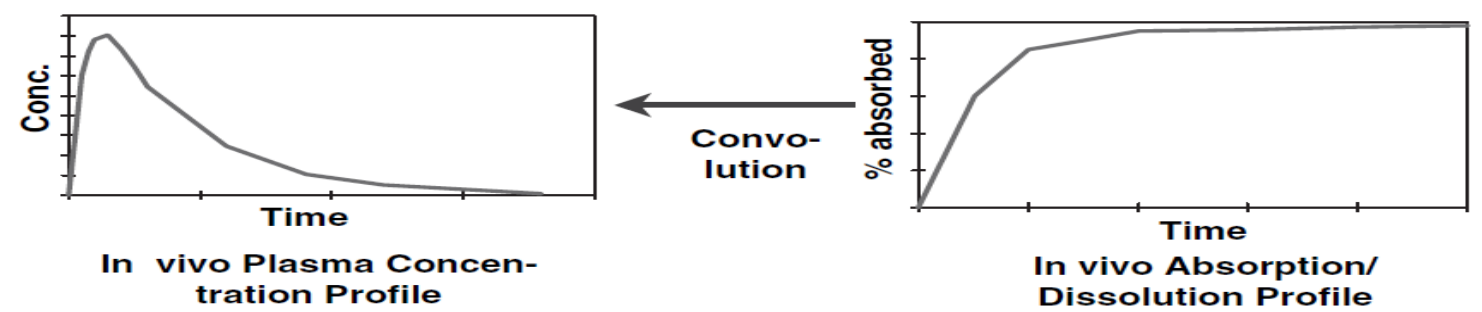

1 step

Dissolution Profile
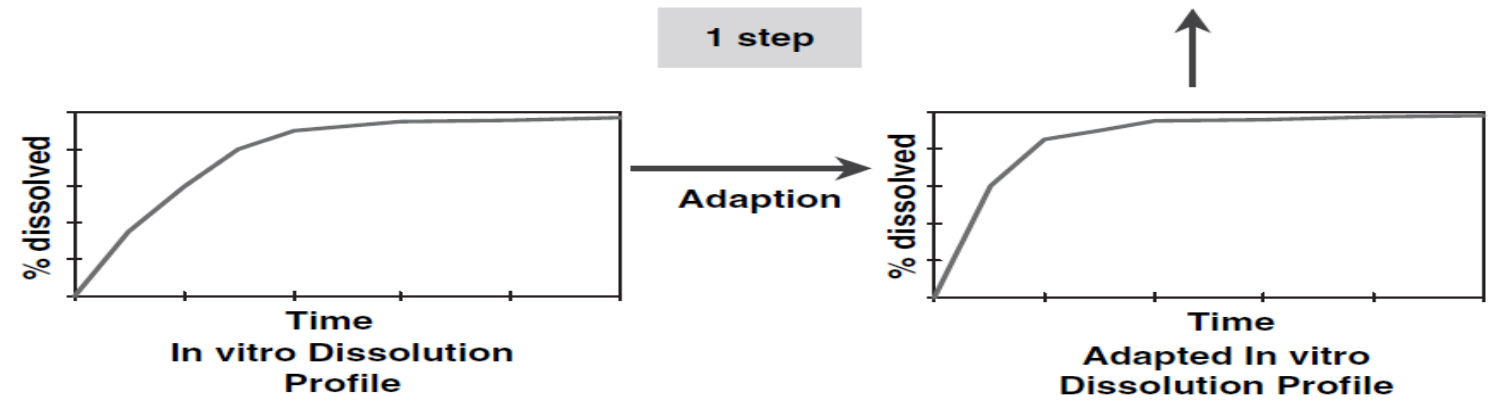

Figure 5. The One-Step Procedure of a Convolution. 
established by comparing the mean in vitro dissolution time to either mean residence time (MRT-in vivo) or the mean dissolution time in vivo of the product. Level B correlation is not point to point correlation. That is why it never replicates the in vivo plasma level actually.

IVIVC at "Level C" It ascertains a single point relationship between in vitro dissolution parameter and a pharmacokinetic parameter. Any dissolution time point is generally compared (e.g., $\mathrm{t}_{90 \%}$ ) to one pharmacokinetic parameter (like AUC, $\mathrm{t}_{\max }$, and $\mathrm{C}_{\max }$ ). This is a very weak level of correlation, and has limited practicability in forecasting in vivo drug performance. Correlations at Level $\mathrm{C}$ means a lot during early phases of development of formulation.

IVIVC at "Multiple Level C" This relationship is almost equivalent to level $\mathrm{A}$, and can be employed as a substitute to the Level A correlation. It relates relevant pharmacokinetic parameter to the drug's quantity dissolved in vitro at respective time points. It can be employed to defend biowaiver(s) if the correlation is established over the entire dissolution contour with one or more pharmacokinetic parameters of significance. If such correlation is possible, then "Level A" correlation is also possible.

IVIVC at Level D: It is a nonparametric rank order correlation between the in vitro dissolution parameter and an in vivo pharmacokinetic parameter. It is usually based on ordinal (but not quantitative) data, thus considered to be the weakest correlation.

Thus IVIVC is defined as ${ }^{12,13}$

United State Pharmacopoeia (USP) definition: The establishment of a sensible relationship between a physiological performance, or a parameter derived from a physiological performance produced by a dosage form, and a distinctive physicochemical performance or property of the same dosage form (US Department 1995).
Food and Drug Administration (FDA) definition: It is a predictive mathematical model relating the relationship between an in vitro performance parameter of a dosage form and it's in vivo response parameter. Generally, the in vitro performance parameter is the rate and extent of drug dissolution or release and the in vivo response is the drug concentration in plasma or quantity of drug absorbed (Center for Drug Evaluation, USFDA 1997).

\section{IVIVC MODEL VALIDATION ${ }^{14,15}$}

For IVIVC, minimum 12 individual dosage units from every formulation batch have to be tested for their dissolution profiles. Bioavailability studies should be completed with that number of subjects which is sufficient to demonstrate the performance of the test formulation adequately. BCS i.e. Biopharmaceutical Classification System is useful to decide the condition under which the predictable IVIVC is expected.

The IVIVC is developed with an aim to predict the plasma conc. time profile. It is, thus very important to judge the predictive concert by calculating its prediction error. Based on the proposed application of the IVIVC and the drug's therapeutic index justification of internal /external predictability may be is done.

It is an estimation of predictive performance of the model proposed during strategic development of the correlation. Prediction error is evaluated considering the proposed application of IVIVC and drug's therapeutic index. Since, IVIVC could be used to anticipate in vivo performance in case of any future change in the formulation, external predictability is essential. External predictability depends on the supplementary data sets (strategic development of IVIVC step 2) and internal predictability depends on initial data of step 1 of strategic development of IVIVC.

Table 1. IVIVC Probabilities for Immediate Release Products.

\begin{tabular}{|c|c|c|c|c|}
\hline CLASS & Solubility & Permeability & $\begin{array}{l}\text { Absorption } \\
\text { rate controlling } \\
\text { parameter }\end{array}$ & For immediate release product \\
\hline I & High & High & Gastric emptying & $\begin{array}{l}\text { IVIVC may be expected; if dissolution rate is slower than gastric } \\
\text { emptying rate, otherwise limited or no correlations. }\end{array}$ \\
\hline II & Low & High & Dissolution & $\begin{array}{l}\text { IVIVC may be expected; if in vitro rate of dissolution is } \\
\text { equivalent to in vivo dissolution rate (except when the dose is } \\
\text { very high). }\end{array}$ \\
\hline III & High & Low & Permeability & Absorption (Permeability) is slow and difficult to establish IVIVC. \\
\hline IV & Low & Low & Case to Case & Limited / no IVIVC is expected. \\
\hline
\end{tabular}

Evaluation of Predictability of IVIVC 
Prediction error ( $\%$ P.E) $\mathrm{C}_{\text {max }}$ and AUC can be calculated as-

$$
\% \text { P.E }=\frac{(\text { observed-predicted }) \times 100}{\text { Observed }}
$$

\section{Internal Predictability}

PDE i.e. Percent Prediction Error (average) of $\leq 10 \%$ establishes predictability. Percent Prediction Error (PDE) for any formulation should not be $>15 \%$. Non compliance with these criteria depicts inconclusive internal predictability and external predictability should be performed.

\section{External Predictability}

Percent Predictability Error $\leq 10 \%$ for $\mathrm{C}_{\max }$ and AUC sets up the external predictability. Percent predictability error between $10-20 \%$ shows inconclusive predictability. In such cases further study is demanded using additional data sets. Percent predictability error $>20 \%$ generally indicates poor predictability, and has to be justified.

\section{IVIVC Model Application ${ }^{16,17}$}

The development and validation of In vivo In vitro correlation (IVIVC) is even more imperative stage of product (new / generic) optimization. It is a linear correlation between in vitro characteristics and a biological parameter $\left(\mathrm{C}_{\max }, \mathrm{t}_{\max }, \mathrm{AUC}\right)$.

Optimization may require modification formulation composition, manufacturing method, required equipments or even in batch size. If these changes are made, in vivo bioavailability study in healthy human volunteers may be required to prove bioavailability of newly developed dosage form with the earlier one. These facts halt the marketing of new formulation with added cost. Hence, it would be inevitable to build up in vitro test that represents bioavailability. Thus this is main point of developing and establishing IVIVC. Once established it serves as proxy to in vivo bioavailability and props up bio waivers. Since IVIVC includes institution of relationship between in vivo parameter (bioavailability) to in vitro parameter (dissolution); it can also be utilized to ascertain dissolution specifications and/or authenticate/validate the dissolution testing methods. It also assists as quality control parameter for certain SUPAC i.e. scale up and post approval changes. Moreover there must be some in vitro means to assure batch to batch consistency during large scale manufacturing. After successful establishing IVIVC; dissolution method used in such establishment used as routine quality control test as a measure of physiological performance of drug product.

\section{CONCLUSION}

While FDA guidelines for establishment of IVIVC are applicable to oral dosage forms; these principles can be used in design and development of non oral dosage forms also. IVIVC's are applicable for granting the bio-waivers, deciding dissolution specifications, setting release rate specifications. A prophetic correlation of level A facilitates the process of proposal as biowaiver formulation. This is so, as the in vitro dissolution substitutes the in vivo bioavailability of the formulation. Therefore, a meaningful and predictive correlation can be a useful tool both in product development and regulatory decision making.

\section{ACKNOWLEDGEMENT}

None declared.

\section{CONFLICT OF INTEREST}

Nil

\section{REFERENCES}

1. U.S. Department of health and human services. Guidance for industrial. Extended release oral dosage forms: development, evaluation and application of in vitro in vivo correlations. Food and drug administration, Center for Drug Evaluation and Research (CDER). Rockville, MD. September; 1997.

2. The united stated pharmacopeia. in vitro in vivo evaluation of dosage forms. Rockville, MD. (23 $\left.{ }^{\text {th }}\right) ;$ 1995. 1824-929.

3. Young D, Devane JG, Butler J. (Eds.). In vitro In vivo correlations. New York: plenum press; 1997.

4. Extended release oral dosage forms: Development, evaluation and application of in vitrolin vivo correlations; Guidance for industry; US FDA, CDER, U.S. Government Printing Office: Washington DC; Sept 1997.

5. Singh B, Mohapatra A, Bandyopadhyay S, kapil R. Endeavoring biowaivers using BCS and IVIVC. The Pharma Review. 2010; 86-93.

6. Chilukuri DM, Sunkara G. IVIVC: An Important Tool in the Development of Drug Delivery Systems. Drug Deliv Technol; 2003. 3-4.

7. Modi NB, Lam A, Lindemulder E, Wang B, Gupta SK. Application of in vitroin vivo correlation (IVIVC) in setting formulation release specifications. Biopharm Drug Dispos. 2000; 21(8): 321-6.

8. United States Pharmacopoeia. in vitro and in vivo Evaluations of Dosage Forms, 27th edition, Revision, Mack Publishing Co., Easton, PA; 2004.

9. Jaber Emami. in vitro - in vivo Correlation: From Theory to Applications. J Pharm Pharm Sci. 2006; 9(2): 169-89.

10. Venkata Raman S. Uppoor. Regulatory perspectives on in vitro (dissolution)/in vivo (bioavailability) correlations. J Control Release. 2001; 72(1): 127-32.

11. Leon Shargel SW. Pong ABC, Yu. Pharmacokinetics of oral absorption. In L. Shargel Editor. Applied Bio pharmaceutics and Pharmacokinetics by. McGraw Hill, New York; 2005. 161-84.

12. The United States Pharmacopeia. in vitro and in vivo evaluation of dosage forms. (1088). 23rd ed. Rockville: U.S. Pharmacopeial Convention; 1995. 1924-9.

13. Center for Drug Evaluation and Research (CDER). Extended release solid dosage forms: development, evaluation and application of in vitrolin vivo correlations. US Department of Health and Human Services. Food and Drug Administration, Guidance for the Industry; Sep 1997. 
14. FDA Guidance for Industry. Extended release oral dosage forms: development, evaluation and applications of in vitrolin vivo correlations; Sep 1997.

15. Uppoor R. Evaluation of predictability of in vivo in vitro correlations, SUPAC-MR/IVIVC guidance FDA training program manual. Center for Drug Evaluation and Research. Food and Drug Administration; July 1997. 97-110.
16. Marroum PJ. Regulatory examples: dissolution specifications and bioequivalence product standards. Scientific Foundations for Regulating Drug Product Quality. Alexandria, VA: AAPS Press; 1997. 305-19.

17. Marroum PJ. Role of in vivo-in vitro correlations in setting dissolution specifications. Am Pharm Rev. 1999; 2: 39-42. 